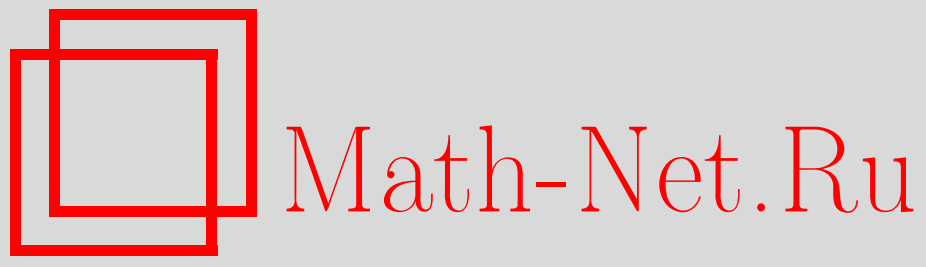

А. Б. Антоневич, Когерентная локальная гиперболичность линейного расширения и существенные спектры оператора взвешенного сдвига на отрезке, Функи. анализ и его прил., 2005, том 39, выпуск 1, 11-26

DOI: https://doi.org/10.4213/faa28

Использование Общероссийского математического портала MathNet.Ru подразумевает, что вы прочитали и согласны с пользовательским соглашением

http://www.mathnet.ru/rus/agreement

Параметры загрузки:

IP : 54.174 .149 .18

26 апреля 2023 г., 14:10:03

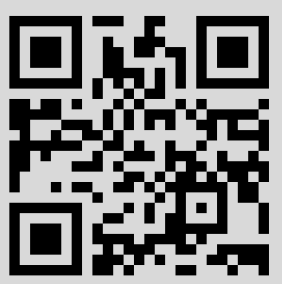


Функииональньй анализ и его приложения

2005, т. 39, вып. 1, с. 11-26

УДК 517.9

\title{
Когерентная локальная гиперболичность линейного расширения и существенные спектры оператора взвешенного сдвига на отрезке
}

\author{
(c) 2005. А. Б. Антоневич
}

\section{$\S 1$. Введение}

Пусть $X$ есть отрезок $[0,1]$ и $\alpha: X \rightarrow X-$ диффеоморфизм, имеющий только две неподвижные точки, 0 и 1 . В пространстве вектор-функций $L_{p}[0,1], 1 \leqslant p \leqslant$ $\infty$, со значениями в $\mathbb{C}^{m}$ рассмотрим оператор взвешенного сдвига вида

$$
B u(x)=a_{0}(x) u\left(\alpha^{-1}(x)\right), \quad x \in X,
$$

где $a_{0}(x)$ - невырожденная непрерывная матричнозначная функция на $X$.

Операторы вида (1) составляют один из конкретных классов общих операторов взвешенного сдвига. Линейный ограниченный оператор $B$, действующий в банаховом пространстве $F(X)$ функций или вектор-функций на произвольном множестве $X$ (в более общей ситуации - в пространстве сечений векторного расслоения над $X)$, называют оператором взвешенного сдвига (OBC), если он может быть представлен в виде

$$
B u(x)=a_{0}(x) u(\phi(x)), \quad x \in X,
$$

где $\phi: X \rightarrow X-$ некоторое отображение, а $a_{0}(x)$ - скалярная или матричнозначная функция на $X$. В (1) мы предполагаем, что отображение $\phi=\alpha^{-1}$ обратимо, случай необратимого $\phi$ имеет свои особенности, которые мы здесь не обсуждаем.

Общие ОВС, порожденные ими операторные алгебры и связанные с ними функциональные уравнения вида

$$
u(x)-a_{0}(x) u(\phi(x))=v(x),
$$

в частности так называемое гомологическое уравнение

$$
u(x)-u(\phi(x))=v(x),
$$

изучались многими авторами в различных функциональных пространствах как самостоятельный объект и в связи с различными приложениями (теорией динамических систем, интегро-функциональными, дифференциально-функциональными, функциональными и разностными уравнениями, автоморфизмами и эндоморфизмами банаховых алгебр, нелокальными краевыми задачами, неклассическими краевыми задачами для уравнения колебаний струны, общей теорией операторных алгебр и др.) [1-15].

Свойства ОВС зависят в первую очередь от динамики отображения $\phi$, т. е. поведения траекторий точек при действии итераций этого отображения. В рассматриваемом конкретном случае диффеоморфизмов отрезка картина поведения траекторий отображения наиболее проста. Поэтому ОВС, порожденные та- 
кими диффеоморфизмами $\alpha$, обычно служат модельными примерами: многие результаты были получены сначала для ОВС вида (1), а затем были перенесены на более общие ситуации. Отметим также, что именно такие отображения возникают в ряде приложений и, например, в теории сингулярных интегрофункциональных уравнений это один из основных объектов исследования [15].

Задача описания спектра общих ОВС в случае обратимого отображения $\phi$ в классических пространствах принципиально решена в достаточной общности; для этого используются обычно два подхода - гиперболический, использующий связи операторов вида (2) со свойством гиперболичности ассоциированного линейного расширения динамической системы, и траекторный, базирующийся на связи с семейством операторов, порожденных сужениями на траектории динамической системы (см. §2).

Наряду с получением условий обратимости представляют интерес тонкие свойства оператора $I-B$ в случае, когда он необратим, такие, например, как замкнутость образа этого оператора (вопрос о замкнутости возникает в ряде приложений) или нормальная, фредгольмова или полуфредгольмова разрешимость в соответствующем пространстве функционального уравнения (3) и гомологического уравнения (4). Ранее такие свойства операторов вида $I-B$ изучались только в пространствах скалярных функций и для специальных классов отображений $[11,12,14]$.

Напомним, что оператор $A$ называется $F^{+}$-оператором ( $F^{-}$-оператором), если его образ $\operatorname{Im} A$ замкнут, а его ядро $\operatorname{Ker} A$ (ядро сопряженного оператора) конечномерно. $F^{+}$-операторы и $F^{-}$-операторы называют полуфредгольмовыми.

В спектральной терминологии вопросы об указанных свойствах оператора $I-B$ суть вопросы о принадлежности числа $\lambda=1$ одному из существенных спектров оператора $B$ в смысле приведенных ниже определений. Для линейного ограниченного оператора $A$ в банаховом пространстве рассматриваются следующие подмножества его спектра $\sigma(A)$, называемые существенными сnектрами:

$$
\begin{aligned}
\sigma_{G}(A) & =\{\lambda: \overline{\operatorname{Im}(\lambda I-A)} \neq \operatorname{Im}(\lambda I-A)\}-\text { спектр Гольдберга, } \\
\sigma_{K}(A) & =\{\lambda: \lambda I-A \text { не полуфредгольмов }\}-\text { спектр Като, } \\
\sigma_{F^{+}}(A) & =\left\{\lambda: \lambda I-A \text { не } F^{+} \text {-оператор }\right\}-F^{+} \text {-спектр, } \\
\sigma_{F}(A) & =\{\lambda: \lambda I-A \text { не фредгольмов }\}-\text { фредгольмов спектр. }
\end{aligned}
$$

Заметим, что в литературе встречаются другие названия для указанных спектров и выделяются другие виды существенных спектров.

В данной работе впервые получено полное описание указанных существенных спектров для ОВС вида (1) в пространствах $L_{p}[0,1]$ вектор-функций. Основным результатом, качественно новым по сравнению с известными ранее, является выявление свойств ассоциированного линейного расширения, отвечающих за тонкие спектральные свойства оператора $B$, и в первую очередь свойства когерентной локальной гиперболичности линейного расширения.

Подход, использованный в настоящей работе, представляет собой синтез гиперболического и траекторного подходов. В случае пространства непрерывных функций близкий результат для модельных операторов был анонсирован в [10]. 


\section{§2. Гиперболический и траекторный подходы к исследованию спектра в случае ОВС вида (1)}

В пространстве $L_{p}[0,1]$ оператор $T u(x)=\alpha^{\prime}\left(\alpha^{-1}(x)\right)^{-1 / p} u\left(\alpha^{-1}(x)\right)$ является изометрическим обратимым оператором. Поэтому оператор (1) в пространстве $L_{p}[0,1]$ более удобно записывать в виде $B=a T$, где $a(x)=\alpha^{\prime}\left(\alpha^{-1}(x)\right)^{1 / p} a_{0}(x)$ есть так называемый приведенный коэффициент.

2.1. Гиперболический подход. Ассоциированное линейное расширение отображения $\alpha$ строится следующим образом.

Рассмотрим произведение $E=X \times \mathbb{C}^{m}$ как векторное расслоение над $X$ с естественной проекцией; его слоем над точкой $x \in X$ является множество

$$
E_{x}=\left\{(x, \xi): \xi \in \mathbb{C}^{m}\right\}=\{x\} \times \mathbb{C}^{m} .
$$

По оператору $B$ зададим отображение $\beta: E \rightarrow E$ формулой

$$
\beta(x, \xi)=(\alpha(x), a(x) \xi), \quad x \in X, \xi \in \mathbb{C}^{m} .
$$

Отображение $\beta$ непрерывно и отображает слой $E_{x}$ на слой $E_{\alpha(x)}$ линейно. Это означает, что $\beta$ является линейным расширением отображения $\alpha$ (см., напримep, [5]).

По функции $a(x)$ строится так называемый коцикл $a(x, n)$, порожденный коэффициентом $a: a(x ; n)=a(x) a(\alpha(x)) \cdots a\left(\alpha_{n-1}(x)\right)$ при $n>0, a(x ; n)=$ $\left[a\left(\alpha_{n-1}(x)\right)\right]^{-1}\left[a\left(\alpha_{n-2}(x)\right)\right]^{-1} \cdots[a(x)]^{-1}$ при $n<0, a(x, 0)=I$.

Тогда для степеней, т. е. итераций, отображения $\beta$ имеем

$$
\beta^{n}(x, \xi)=\left(\alpha^{n}(x), a(x ; n) \xi\right), \quad n \in \mathbb{Z} .
$$

Будем считать, что норма вектора $\xi \in \mathbb{C}^{m}$ задана стандартным скалярным произведением $\langle\xi, \eta\rangle$, и обозначать ее через $\|\xi\|$ или $|\xi|$. Положим $\|(x, \xi)\|=|\xi|$.

Напомним, что подмножество $K$ расслоения $E$ называется векториальным [5], если для любого $x$ множество $K_{x}:=K \cap E_{x}$ является векторным подпространством в $E_{x}$; векториальное подмножество, у которого подпространства $K_{x}$ непрерывно зависят от $x$, называется подрасслоением.

Подрасслоение $E^{s}$ расслоения $E$ называется устойчивым в положителъном направлении (неустойчивым в положительном направлении) для линейного расширения $\beta$, если оно инвариантно относительно действия $\beta$ и существуют постоянная $\gamma_{s}, 0<\gamma_{s}<1\left(\gamma_{u}, 0<\gamma_{u}<1\right)$, и постоянная $C>0$, при которых выполнено неравенство

$$
\begin{array}{cl}
\left\|\beta^{n}(x, \xi)\right\| \leqslant C \gamma_{s}^{n}\|\xi\|, & x \in[0,1],(x, \xi) \in E^{s}, n=1,2, \ldots \\
\left(\left\|\beta^{n}(x, \xi)\right\| \geqslant C \gamma_{u}^{-n}\|\xi\|,\right. & \left.x \in[0,1],(x, \xi) \in E^{u}, n=1,2, \ldots\right) .
\end{array}
$$

Линейное расширение называется гиперболическим, если оно разлагается в прямую сумму Уитни устойчивого и неустойчивого подрасслоений [5].

Динамическим спектром $\sigma(\beta)$ линейного расширения $\beta$ называется множество чисел $\lambda$, для которых линейное расширение $\beta_{\lambda}$ из спектрального семейства линейных расширений

$$
\beta_{\lambda}(x, \xi)=\left(\alpha(x), \lambda^{-1} a(x) \xi\right), \quad \lambda \in \mathbb{C}, \lambda \neq 0,
$$

не является гиперболическим. 
Линейный оператор $A$ называется гиперболическим, если его спектр не пересекается с единичной окружностью.

ПРЕДЛОЖЕНИЕ 1 (теорема о гиперболичности). Оператор $I-B$ в пространстве $L_{p}[0,1]$, где $B$ - оператор вида (1), обратим тогда и только тогда, когда ассочиированное линейное расширение $\beta$ является гиперболическим; при выполнении этого условия оператор $B$ является гиперболическим.

СледСтвиЕ. Спектр оператора $B$ совпадает с динамическим спектром линейного расширения $\beta$ и состоит не более чем из $m$ непересекающихся колеи, с иентром в точке 0 .

2.2. Траекторный подход базируется на следующей конструкции. Пусть $l_{p}$ есть пространство двусторонних последовательностей $u=(u(k)), k \in \mathbb{Z}$, $u(k) \in \mathbb{C}^{m}$, векторов из $\mathbb{C}^{m}$ с нормой $\|u\|=\left(\sum_{k}|u(k)|^{p}\right)^{1 / p}$. Обозначим через $B(\tau), \tau \in X$, дискретный оператор взвешенного сдвига, действующий в $l_{p}$ по формуле

$$
(B(\tau) u)(k)=a\left(\alpha^{k}(\tau)\right) u(k-1) .
$$

Оператор $B(\tau)$ построен с помощью сужения коэффициента $a$ на траекторию точки $\tau$, что поясняет название «траекторный подход».

ПРЕДЛОЖКНИЕ 2 (теорема о дискретизации). Оператор $I-B$ обратим тогда u только тогда, когда обратимы все операторы $I-B(\tau), \tau \in X$.

Так как $T(I-B(\tau)) T^{-1}=I-B(\alpha(\tau))$, операторы $I-B(\tau)$ и $I-B((\alpha(\tau))$ обратимы одновременно и нормы их обратных совпадают. Поэтому, если зафиксировать точку $t \in(0,1)$ и отрезок $\Theta=[\alpha(t), t]$, достаточно потребовать обратимость операторов $I-B(\tau)$ только при $\tau \in \Theta$.

СЛЕДСТВИЕ. Имеет место равенство

$$
\sigma(B)=\bigcup_{\tau \in \Theta} \sigma(B(\tau))
$$

Относительно истории вопроса и развития этих подходов см. монографии $[3,9,13,15]$ и обзорные статьи $[4,8]$.

\section{§3. Когерентная локальная гиперболичность}

Рассмотрим некоторые ослабления свойства гиперболичности линейного расширения. Для определенности будем считать, что $\alpha(x)<x$ для $0<x<1$, и тогда точка 0 является притягивающей - траектория любой точки $x \neq 1$ стремится к точке 0 (случай $\alpha(x)>x$ может быть рассмотрен аналогично).

ОПРЕДЕЛЕНИЕ 1 . Подрасслоение $E^{s, 0}$ расслоения $E$, определенное на окрестности $[0, \delta]$ притягивающей точки 0, называется (локально) устойчивым в положительном направлении (неустойчивым в положительном направлении) в точке 0 для линейного расширения $\beta$, если оно инвариантно при действии $\beta$ и существуют постоянные $\gamma_{s}, 0<\gamma_{s}<1\left(\gamma_{u}, 0<\gamma_{u}<1\right)$, и $C>0$, при которых для всех $x$ из окрестности $[0, \delta]$ выполнено неравенство

$$
\begin{array}{ll}
\left\|\beta_{n}(x, \xi)\right\| \leqslant C_{s} \gamma_{s}^{n}\|\xi\|, & (x, \xi) \in E^{s, 0}, n=1,2, \ldots \\
\left(\left\|\beta_{n}(x, \xi)\right\| \geqslant C_{u} \gamma_{u}^{-n}\|\xi\|,\right. & \left.x \in[0, \delta],(x, \xi) \in E^{u, 0}, n=1,2, \ldots\right) .
\end{array}
$$


Линейное расширение $\beta$ будем называть локалъно гиперболическим в точке 0 , если $E$ разлагается в прямую сумму устойчивого и неустойчивого подрасслоений в некоторой окрестности точки 0 .

Аналогично определяются устойчивое и неустойчивое подрасслоения в отрицательном направлении в окрестности отталкивающей точки 1 - как устойчивое и неустойчивое в положительном направлении для обратного отображения $\beta^{-1}$ - и локальная гиперболичность в точке 1.

Любое подрасслоение, определенное и инвариантное в окрестности точки 0 , может быть однозначно продолжено с окрестности до инвариантного подрасслоения на интервале $[0,1)$. Если исходное подрасслоение устойчиво (или неустойчиво) в положительном направлении, то продолженное подрасслоение также будет устойчивым (неустойчивым) на любом другом отрезке $[0, \delta]$ при $\delta<1$ и соответствующее неравенство выполнено с той же постоянной $\gamma_{s}$, но, вообще говоря, с другой постоянной $C=C(\delta)$.

В общем случае инвариантное подрасслоение (даже устойчивое) не может быть продолжено с окрестности точки 0 до непрерывного инвариантного подрасслоения на всем отрезке $[0,1]$. Заметим, что даже в случае, когда такое продолжение существует, оно может не быть глобально устойчивым, так как может оказаться, что числа $C(\delta)$ неограниченно возрастают при $\delta \rightarrow 1$.

Если линейное расширение гиперболическое, то подрасслоение, устойчивое (неустойчивое) в положительном направлении, является неустойчивым (устойчивым) в отрицательном направлении. При локальной гиперболичности такое положение не имеет места и все четыре подрасслоения могут быть различными. При этом локально устойчивые подрасслоения определены однозначно, но существует много различных локально неустойчивых подрасслоений.

Пусть линейное расширение $\beta$ локально гиперболично в точках 0 и 1 ; пусть $E^{s, 0}$ и $E^{s, 1}$ суть соответствующие устойчивые подрасслоения, которые, согласно сделанному выше замечанию, мы можем считать определенными на $[0,1)$ и $(0,1]$ соответственно. Взаимное расположение этих подрасслоений будем описывать с помощью функции

$$
d(\tau)=\operatorname{dim} V_{\tau}, \quad \text { где } V_{\tau}:=E_{\tau}^{s, 0} \cap E_{\tau}^{s, 1}, \tau \in(0,1) .
$$

Так как в силу инвариантности подрасслоений имеем $d(\tau)=d(\alpha(\tau))$, эту функцию достаточно рассматривать на промежутке $\Theta=[\alpha(t), t]$, где $t \in(0,1)$ есть некоторая произвольно зафиксированная точка. Отметим, что функция $d(\tau)$ может быть разрывной, но она полунепрерывна сверху. Если $d(\tau)$ непрерывна, то она постоянна и подпространства $V_{\tau}$ задают подрасслоение $V$ расслоения $E$ над $\Theta$, а в противном случае множество $V=E^{s, 0} \cap E^{s, 1}$ является только векториальным множеством.

Сопряженный оператор $B^{*}$, действующий при $p<\infty$ в пространстве $L_{q}[0,1]$, где $1 / p+1 / q=1$, имеет вид

$$
B^{*} u(x)=a_{0}^{*}(\alpha(x)) \alpha^{\prime}(x) u(\alpha(x),
$$

где $a^{*}(x)$ есть эрмитово сопряженная матрица к $a(x)$; соответствующее ему линейное расширение, действующее по формуле

$$
\beta^{*}(x, \xi)=\left(\alpha^{-1}(x),\left(a^{*}(\alpha(x)) \xi\right), \quad x \in X, \xi \in \mathbb{C}^{m},\right.
$$


называется сопряженным к линейному расширению $\beta[5]$. В случае $p=\infty$ оператор $B$ является сопряженным к соответствующему оператору в пространстве $L_{1}[0,1]$. Изучаемые свойства операторов имеют место одновременно для оператора и сопряженного (с заменой свойства $F^{+}$на $F^{-}$), и поэтому достаточно рассмотреть случай $p<\infty$.

Как следует из леммы 1 , локальная гиперболичность расширений $\beta$ и $\beta^{*}$ имеет место одновременно; для $\beta^{*}$ определена аналогичная функция $d^{*}(\tau)$ и справедливо равенство $d(\tau)+m=d^{*}(\tau)+\operatorname{dim} E_{\tau}^{s, 0}+\operatorname{dim} E_{\tau}^{s, 1}$.

ОПРЕДЕЛЕНИЕ 2. Будем говорить, что линейное расширение $\beta$ когерентно локально гиперболично (или что локальные гиперболические структуры в точке 0 и в точке 1 когерентны), если $d(\tau)=$ const.

Линейное расширение $\beta$ будем называть плюс-полугиперболическим (минусnолугиперболическим), если оно локально гиперболическое и $d(\tau)=0\left(d^{*}(\tau)\right.$ $=0)$.

Обозначим через $\lambda_{j}(0)$ собственные значения матрицы $a(0)$, и пусть $m(0)$ есть количество (с учетом кратности) собственных чисел, удовлетворяющих условию $\left|\lambda_{j}(0)\right|<1$. Аналогично, пусть $\lambda_{j}(1)$ суть собственные значения матрицы $a(1)$ и $m(1)$ - число собственных значений, удовлетворяющих условию $\left|\lambda_{j}(1)\right|<1$.

ЛЕмма 1. Линейное расширение $\beta$ является локально гиперболическим 8 точке 0 (в точке 1) тогда и только тогда, когда выполнено условие $\left|\lambda_{j}(0)\right| \neq 1$ (соответственно $\left|\lambda_{j}(1)\right| \neq 1$ ) для всех $j ;$ при этом размерность устойчивого подрасслоения $E^{s, 0}\left(E^{s, 1}\right)$ есть $m(0)$ (соответственно $\left.m-m(1)\right)$.

Заметим, что утверждение леммы 1 является одним из простейших вариантов теорем об устойчивости свойства гиперболичности. Из этой леммы вытекает возможность так называемой «факторизации со сдвигом» коэффициента $a$. Пусть числа $r_{j}(0), r_{j}(0)<r_{j+1}(0), j=1,2, \ldots, l(0)$, суть все различные модули собственных значений матрицы $a(0)$, занумерованные в порядке возрастания, и пусть $m_{j}(0)$ - количество (с учетом кратности) чисел $\lambda_{k}(0)$, удовлетворяющих неравенству $\left|\lambda_{k}(0)\right| \leqslant r_{j}(0)$.

ЛЕмма 2. Для непрерывной матрищы-функиии а существует определенная на $[0,1)$ непрерьвная невырожденная матрииа-функиия $S(x)$, такая, что

$$
S(x) a(x) S^{-1}(\alpha(x))=d(x), \quad a(x)=S^{-1}(x) d(x) S(\alpha(x)),
$$

где матрица $d(x)$ является блочно-диагональной с блоками $d_{j}(x)$ размерности $m_{j}(0)-m_{j-1}(0)$, такими, что модули всех собственных значений матриць $d_{j}(0)$ равны $r_{j}(0)$.

СлЕДСТВиЕ 1. Укрупнив блоки, матрицу $d$ можно представить в блочнодиагональном виде $\operatorname{diag}\left\{d^{s}(x), d^{n}(x), d^{u}(x)\right\}$, где у указанных блоков модули собственных значений в точке 0 соответственно менъше 1, равны 1 и больше 1.

Установим более детально связь между оператором $B$ и семейством операторов $B(\tau), \tau \in \Theta$.

Заметим, что пространство $L_{p}(\Theta \times \mathbb{Z})$ с нормой

$$
\|u\|=\left(\sum_{k \in \mathbb{Z}} \int_{\Theta}|u(\tau, k)|^{p} d \tau\right)^{1 / p}
$$


может быть представлено как пространство $L_{p}\left(\Theta ; l_{p}\right)$ измеримых функций $U(\tau)$ на $\Theta$ со значениями в $l_{p}$ и как пространство $l_{p}\left(L_{p}(\Theta)\right)$ двусторонних последовательностей $U=(U(k))$ с элементами из $L_{p}(\Theta)$.

ЛЕмма 3. 1. Отображсение $\Pi: u \rightarrow U$, где $U(\tau, k)=\left(\alpha^{\prime}(\tau, k)^{1 / p} u\left(\alpha^{k}(\tau)\right)\right)$, $k \in \mathbb{Z}, \tau \in \Theta, u \alpha^{\prime}(x, k)$ - коиикл, порожденный производной $\alpha^{\prime}(x)$, определяет изометрический изоморфизм между пространством $L_{p}[0,1]$ и пространством $L_{p}(\Theta \times \mathbb{Z}) \sim L_{p}\left(\Theta ; l_{p}\right)$. При этом изоморбизме оператор $B$ переходит в оператор $\widetilde{B}$, действующий как оператор умножения на операторнозначную функиию $B(\tau)$, т.е. по формуле

$$
(\widetilde{B} U)(\tau)=B(\tau) U(\tau) .
$$

2. Операторнозначная функиия $B(\tau)$ непреръвна по операторной норме на $\Theta$.

В дальнейшем будет удобно отождествлять оператор $\widetilde{B}$ с $B$.

Операторы $B(\tau)$ действуют в пространствах последовательностей и более доступны для исследования. Мы воспользуемся следующим известным утверждением.

ПРЕДЛОЖЕНИЕ 3. Оператор $I-B(\tau)$ фредголъмов тогда и только тогда, когда $\left|\lambda_{j}(0)\right| \neq 1$ u $\left|\lambda_{j}(1)\right| \neq 1$, m. е. когда выполненъ условия локальной гиперболичности в точках 0 и 1 .

Множество $K=\{(\tau, u): u \in \operatorname{Ker}(I-B(\tau))\}$ есть векториальное подмножество в $\Theta \times l_{p}$. Зададим на нем метрику, индуцированную из произведения:

$$
\rho\left(\left(\tau_{1}, u_{1}\right),\left(\tau_{2}, u_{2}\right)\right)=\left|\tau_{1}-\tau_{2}\right|+\left\|u_{1}-u_{2}\right\|_{l_{p}} .
$$

Определенное выше векториальное множество $V$ будем рассматривать с метрикой, индуцированной из $E$ :

$$
\rho\left(\left(\tau_{1}, \xi_{1}\right),\left(\tau_{2}, \xi_{2}\right)\right)=\left|\tau_{1}-\tau_{2}\right|+\left\|\xi_{1}-\xi_{2}\right\|
$$

ЛЕмма 4. Если выполнены условия локальной гиперболичности в точках 0 $u 1, m o$

(i) для $\tau \in \Theta$ существует линейное биективное отображсение $J(\tau): V_{\tau} \rightarrow$ $\operatorname{Ker}(I-B(\tau))$, такое, что отображсние $J: V \ni(\tau, \xi) \rightarrow(\tau, J(\tau) \xi) \in K$ является гомеоморбизмом между $V$ и $K$, причем это отображсение и его обратное равномерно непрерьвны;

(ii) $\operatorname{dim} \operatorname{Ker}(I-B(\tau))=d(\tau), \operatorname{dim} \operatorname{Ker}\left(I-B^{*}(\tau)\right)=d^{*}(\tau)$.

ДокАзАтЕльство. Любое решение (в пространстве всех последовательностей) однородного уравнения $u(k)-a\left(\alpha^{k}(\tau)\right) u(k-1)=0$ однозначно определяется своим начальным условием, значением $u(0)$, и имеет вид $u(k)=a(\tau, k) u(0)$, где $a(x, k)$ - коцикл, порожденный коэффициентом $a$.

Если $u(0) \in E_{\tau}^{s}(0)$, то эта последовательность экспоненциально убывает при $k \rightarrow+\infty$, а если $u(0) \notin E_{\tau}^{s}(0)$, то указанная последовательность растет на $+\infty$. Аналогично если $u(0) \in E_{\tau}^{s}(1)$, то эта последовательность экспоненциально убывает при $k \rightarrow-\infty$, а если $u(0) \notin E_{\tau}^{s}(1)$, то указанная последовательность растет на $-\infty$. 
Поэтому построенная последовательность $(u(k))$ принадлежит пространству $l_{p}$, т. е. является решением уравнения $(I-B(\tau)) u=0$, тогда и только тогда, когда $u(0) \in V_{\tau}$. Значит, линейный оператор

$$
J(\tau): V_{\tau} \ni \xi \rightarrow(u(k))=(a(\tau, k) \xi) \in \operatorname{Ker}(I-B(\tau))
$$

(ограниченный, поскольку он является отображением конечномерных пространств) является биективным и $d(\tau)=\operatorname{dim} \operatorname{Ker}(I-B(\tau))$.

Аналогично, $d^{*}(\tau)=\operatorname{dim} \operatorname{Ker}\left(I-B^{*}(\tau)\right)$.

Рассмотрим семейство операторов $J^{+}(\tau): E_{\tau}^{s, 0} \rightarrow l_{p}, \tau \in \Theta$, определенное формулой $J^{+}(\tau) \xi=\left(U^{+}(k)\right) \in l_{p}$, где $U^{+}(k)=a(\tau, k) \xi$, если $k \geqslant 0$, и $U^{+}(k)=0$, если $k<0$. Используя приведение матрицы $a(x)$ на $[0,1)$ к блочнодиагональному виду $d(x)=\operatorname{diag}\left\{d_{0}^{s}(x), d_{0}^{u}(x)\right\}$, мы можем рассматривать эти отображения как отображения из одного пространства (типового слоя расслоения $E_{\Theta}^{s, 0}$ ) в пространство $l_{p}$ и получаем, что это семейство операторов непрерывно в смысле нормы, откуда следует равномерная непрерывность отображения $J^{+}:(\tau, \xi) \rightarrow J^{+}(\tau) \xi$ как отображения из $E_{\Theta}^{s, 0}$ в $L\left(\Theta, l_{p}\right)$ с метриками (8) и (9).

Аналогично, семейство операторов, определенное формулой $J^{-}(\tau) \xi=\left(U^{-}(k)\right)$ $\in l_{p}$, где $U^{-}(k)=a(\tau, k) \xi$, если $k<0$, и $U^{-}(k)=0$, если $k \geqslant 0$, задает равномерно непрерывное отображение $J^{-}$из $E_{\Theta}^{s, 1}$ в $L\left(\Theta, l_{p}\right)$.

Отображение $J$ определено на пересечении расслоений $E_{\Theta}^{s, 0}$ и $E_{\Theta}^{s, 1}$, задается там формулой $J=J^{+}+J^{-}$и, следовательно, является равномерно непрерывным.

\section{§4. Основная теорема}

Tеорема 1. Пусть $X=[0,1], \alpha: X \rightarrow X$ есть $C^{1}$-дифбеоморфизм, такой, что $\alpha(0)=0, \alpha(1)=1, \alpha(x) \neq x$ при $0<x<1, B$ - оператор вида (1) в пространстве $L_{p}[0,1]$, где $a_{0}$ - невырожденная непрерывная матрица-функи,я, $\beta$ - ассочиированное с ним линейное расширение вида (5) и $B(\tau), \tau \in \Theta$, дискретные операторы вида (6).

Следующие свойства эквивалентны:

(i) образ оператора $I-B$ замкнут;

(ii) линейное расширение $\beta$ когерентно локально гиперболично;

(iii) все операторы $I-B(\tau), \tau \in \Theta$, фредгольмовы и их ядра имеют одинаковую размерность.

Если выполнены эти условия, то ядро и образ оператора $I-B$ дополняемы.

СлеДСтвие. 1. Oператор $I-B$ является $F^{+}$-оператором тогда и только тогда, когда $\beta$ плюс-полугиперболично; в этом случае $\operatorname{Ker}(I-B)=0 u$ оператор обратим слева.

2. Оператор $I-B$ является $F^{-}$-оператором тогда и только тогда, когда $\beta$ минус-полугиперболично; в этом случае $\operatorname{Im}(I-B)=L_{p}[0,1]$ и оператор обратим справа.

3. Оператор $I-B$ является фредгольмовым тогда и толъко тогда, когда $\beta$ когерентно локально гиперболично и $d(\tau)=d^{*}(\tau)=0$; в этом случае оператор $I-B$ обратим. 
ДоКАЗАТЕЛЬСтво тЕОРемЫ 1. Приведем доказательство для $1<p<\infty$. В случае $p=1$ доказательство содержит некоторые модификации, которые мы опускаем из-за ограниченности размера статьи. Как и выше, достаточно рассмотреть случай, когда выполнено условие $\alpha(x) \leqslant x$.

Эквивалентность условий (ii) и (iii) доказана в лемме 4.

1. Пусть выполнено (iii). Покажем, что образ оператора $I-B$ замкнут. Coгласно лемме 3 , оператор $I-B$ представлен в виде непрерывной операторнозначной функции $I-B(\tau)$, все значения которой являются фредгольмовыми операторами, причем их ядра имеют одинаковую размерность. Так как $d(\tau) \equiv d=$ const, векториальное множество $V$ является подрасслоением расслоения $E$ над $\Theta$. Поскольку над отрезком любое расслоение является тривиальным, можно выбрать ортонормированный базис $\left(e_{j}(\tau)\right), j=1, \ldots, d$, в каждом слое расслоения $V$ над $\Theta$, непрерывно зависящий от $\tau$. Согласно лемме 4 , каждый базисный вектор $e_{j}(\tau)$ в слое $V_{\tau}$ порождает элемент $w_{j}(\tau):=J(\tau) e_{j}(\tau)$ из ядра $\operatorname{Ker}(I-B(\tau))$, причем эти элементы непрерывно зависят от $\tau$ в норме $l_{p}$. Формула

$$
P(\tau) u=\sum_{j=1}^{d}\left\langle u(0), e_{j}(\tau)\right\rangle w_{j}(\tau)
$$

задает ограниченный проектор в $l_{p}$ на $\operatorname{Ker}(I-B(\tau))$, причем это семейство проекторов непрерывно зависит от $\tau$ и ограничено по норме в совокупности.

Поэтому семейство $P(\tau)$ задает ограниченный проектор $P$ в пространстве $L_{p}\left[\Theta, l_{p}\right]$, и этот проектор является проектором на ядро оператора $I-B$. Проектор $P$ порождает разложение в прямую сумму подпространств: $L_{p}\left[\Theta, l_{p}\right]=$ $\operatorname{Ker}(I-B) \oplus \operatorname{Ker} P$. Обозначим через $(I-B)_{P}$ сужение оператора $I-B$ на Ker $P$. Так как $\operatorname{Im}(I-B)=\operatorname{Im}(I-B)_{P}$, достаточно доказать замкнутость последнего множества. Оператор $(I-B)_{P}$ по построению инъективен и для него замкнутость образа эквивалентна выполнению неравенства

$$
\|(I-B) u\| \geqslant C\|u\|, \quad C>0, u \in \operatorname{Ker} P .
$$

Пусть $m(\tau)=\inf \{\|[I-B(\tau)] u\|: u \in \operatorname{Ker} P(\tau),\|u\|=1\}$. Для каждого $\tau$ сужение оператора $I-B(\tau)$ на подпространство $\operatorname{Ker} P(\tau)$ является инъективным оператором с замкнутым образом, откуда следует, что $m(\tau)>0$. Поскольку функция $m(\tau)$ полунепрерывная снизу, она имеет на отрезке $\Theta$ минимум $m\left(\tau^{*}\right):=C>0$. Тогда в силу представления (7) выполнена оценка (11), из которой следует замкнутость образа.

Применяя те же рассуждения к сопряженному оператору, получаем проектор $Q$ на ядро оператора $I-B^{*}$. Тогда оператор $(I-Q)^{*}$ является ограниченным проектором на $\operatorname{Im}(I-B)$, откуда следует дополняемость этого подпространства.

2. Докажем необходимость условия локальной гиперболичности. Пусть образ оператора $I-B$ замкнут. Тогда оператор, индуцированный оператором $I-B$, отображает факторпространство $L_{p}[0,1] / \operatorname{Ker}(I-B)$ на $\operatorname{Im}(I-B)$ биективно и взаимно непрерывно и, значит, существует постоянная $C>0$, такая, что $\|(I-B) u\| \geqslant C\|[u]\|$, где $[u]$ есть порожденный $u$ элемент факторпространства и норма в правой части есть норма в факторпространстве. Предположим, что условие локальной гиперболичности нарушено в точке 0 , и покажем, что тогда 
для любого $\varepsilon>0$ существует функция $u \in L_{p}[0,1],[u] \neq 0$, для которой

$$
\|(I-B) u\| \leqslant \varepsilon\|[u]\|,
$$

что противоречит предыдущему неравенству.

Запишем в силу следствия 2 леммы 2 матрицу $d(x)$ в виде $d(x)=\operatorname{diag}\left\{d^{s}(x)\right.$, $\left.d^{n}(x), d^{u}(x)\right\}$. Такое представление этой матрицы порождает разложение расслоения $E$ в окрестности точки 0 в прямую сумму трех инвариантных относительно действия $\beta$ подрасслоений $E^{s}, E^{n}$ и $E^{u}-$ локально устойчивого, нейтрального и неустойчивого. Перейдя к эквивалентной норме, можем считать, что эти подрасслоения ортогональны.

Пусть $\eta$ есть единичный собственный вектор матрицы $d^{n}(0)$ с собственным значением $\lambda_{\nu}$, где $\left|\lambda_{\nu}\right|=1$. Тогда для $\varepsilon>0$ существует $\delta_{1}>0$, такое, что при $x<\delta_{1}$ выполнено неравенство

$$
\left\|\lambda_{\nu} \eta-d^{n}(x) \eta\right\|<\frac{1}{8} \varepsilon
$$

Пусть $u_{0}(x)=h^{-1 / p} \eta$ при $x \in \Theta, h=t-\alpha(t)$, и $u_{0}(x)=0$ при остальных $x$. Рассмотрим семейство функций, зависящее от параметров $N$ и $N_{1}$ :

$$
u=(N+1)^{-1 / p} \sum_{N_{1}}^{N_{1}+N} \lambda_{\nu}^{-k} T^{-k} u_{0}
$$

Так как носители слагаемых в (14) не пересекаются и оператор $T$ изометрический, то $\|u\|=\left\|u_{0}\right\|=1$.

Выбрав $N_{1}$ достаточно большим, получаем $\alpha^{N_{1}}(t)<\delta_{1}$. Поэтому для $x \in \Theta$ и $k>N_{1}$ имеем $\alpha^{k}(x) \in\left[0, \delta_{1}\right]$ и выполнено (13). Так как

$$
(I-B) u=(N+1)^{-1 / p}\left\{u_{0}+B T^{-N} u_{0}+\sum_{1}^{N-1} \lambda_{\nu}^{k-1}\left[\lambda_{\nu} \eta-d^{n}\left(\alpha^{k}(x)\right) \eta\right]\right\}
$$

получаем оценку $\|(I-B) u\| \leqslant \frac{1}{8} \varepsilon(N+1)^{-1 / p}\left[(N-1)^{1 / p}+\right.$ const $]$ и при достаточно большом $N$ имеем

$$
\|(I-B) u\| \leqslant \frac{1}{4} \varepsilon\|u\| .
$$

Это еще не есть требуемая оценка (12), так как в (12) в правой части стоит меньшая величина - норма в факторпространстве: $\|[u]\|=\inf \{\|u-w\|: w \in$ $\operatorname{Ker}(I-B)\}$.

Покажем, что при достаточно большом $N_{1}$ для построенной функции выполнено $\|[u]\| \geqslant 1 / 4$, откуда следует неравенство (12), что и требовалось.

Неравенство (15) означает, что функция $u$ является «почти собственной» функцией оператора $B$ с собственным значением 1 . Неравенство $\|[u]\| \geqslant 1 / 4$ показывает, что она сильно отличается от настоящих собственных функций. Для получения последнего неравенства проанализируем поведение собственных функций в окрестности нуля.

Так как $w \in \operatorname{Ker}(I-B)$ является сечением расслоения $E$, разложение расслоения $E$ на инвариантные подрасслоения порождает в окрестности нуля разложение $w(x)=w_{s}(x)+w_{n}(x)+w_{u}(x)$, где $w_{s}$ является сечением расслоения $E^{s}, w_{u}$ - сечением расслоения $E^{u}$, а $w_{n}$ - сечением расслоения $E^{n}$. Из неустойчивости расслоения $E^{u}$ следует, что $w_{u}=0$ и в действительности всегда $w=w_{s}+w_{n}$. 
Так как $u$ является по построению сечением расслоения $E^{n}$, то в силу предположения об ортогональности подрасслоений $|u(x)-w(x)|=\left(\left|u(x)-w_{n}(x)\right|^{2}+\right.$ $\left.\left|w_{s}(x)\right|^{2}\right)^{1 / 2} \geqslant\left|u(x)-w_{n}(x)\right|$.

Возможны следующие два случая, причем оба эти случая реализуются в приmepax.

Пусть выполнено условие типа условия Фавара (см. [5]): для любой точки $(x, \xi) \in E^{n}, \xi \neq 0$, траектория $\beta^{k}(x, \xi)$ не стремится к нулю при $k \rightarrow+\infty$. Тогда траектория точки $(\tau, \xi)$ из $E^{n}$ не может порождать элемент ядра оператора $I-B(\tau)-$ соответствующий ряд расходится, а значит, $w_{n}=0$, откуда $\|[u]\|=$ $\|u\|$, и в этом случае утверждение доказано.

Пусть теперь условие типа условия Фавара не выполнено. Тогда могут существовать ненулевые элементы $w$ ядра, являющиеся сечениями нейтрального подрасслоения $E^{n}$, и нужно оценить расстояние от $u$ до таких элементов ядра.

Элементу $(\tau, \xi) \in E^{n}, \tau \in \Theta$, поставим в соответствие последовательность $w(\tau, \xi)$, где $w(\tau, \xi)(k)=a(\tau, k) \xi$ при $k \geqslant 0$ и $w(\tau, k)=0$ при $k<0$. Пусть $S$ есть множество пар $(\tau, \xi) \in E^{n}$, таких, что $w(\tau)$ принадлежит $l_{p}$. Множество $S$ векториально и формула $G(\tau): S_{\tau} \ni \xi \rightarrow w(\tau) \in l_{p}$ задает линейное биективное отображение векторного пространства $S_{\tau}$ на некоторое конечномерное подпространство $W(\tau)$ в $l_{p}$. Если последовательность $w$ принадлежит $\operatorname{Ker}(I-B(\tau))$, то при $k \geqslant 0$ она совпадает с одной из последовательностей из $W(\tau)$.

Рассмотрим последовательность непрерывных функций

$$
\phi_{n}(\tau, \xi)=\left(\sum_{0}^{n}|a(\tau, k) \xi|^{p}\right)^{-1 / p}|\xi|^{-1}, \quad \tau \in \Theta, \xi \in E_{\tau}^{n}, \xi \neq 0 .
$$

Она монотонно возрастает, и в каждой точке существует конечный или бесконечный предел. Тогда $S$ есть множество пар $(\tau, \xi)$, для которых этот предел конечен. Обозначим этот предел через $\phi(\tau, \xi)$. Заметим, что $\phi(\tau, \xi) \neq 0$ при $\xi \neq 0$. По построению имеем

$$
\|G(\tau) \xi\|=\phi(\tau, \xi)\|\xi\|
$$

в частности, при фиксированном $\tau$ функция $\phi(\tau, \xi)$ непрерывна по $\xi$ при $\xi \neq 0$, положительно однородна степени 0 и $\|G(\tau)\|=\max \left\{\phi(\tau, \xi): \xi \in S_{\tau}, \xi \neq 0\right\}$.

При фиксированном $\tau$ последовательность $\phi_{n}(\tau, \xi)$ состоит из непрерывных функций, монотонно возрастает и имеет пределом непрерывную функцию $\phi(\tau, \xi) ;$ по теореме Дини отсюда следует, что эта последовательность сходится равномерно по $\xi$. Поэтому последовательность функций

$$
f_{n}(\tau)=\max \left\{\left[\phi(\tau, \xi)-\phi_{n}(\tau, \xi)\right][\phi(\tau, \xi)]^{-1}: \xi \in K_{\tau}, \xi \neq 0\right\}
$$

на множестве $\Theta$ точечно сходится к нулю. Эти функции измеримы, так как

$$
f_{n}(\tau)=\lim _{m \rightarrow \infty} \max \left\{\left[\phi_{m}(\tau, \xi)-\phi_{n}(\tau, \xi)\right]\left[\phi_{m}(\tau, \xi)\right]^{-1}: \xi \in S_{\tau}, \xi \neq 0\right\} .
$$

По теореме Егорова существует подмножество $\Theta_{1}$ с мерой Лебега $\mu\left(\Theta_{1}\right)>$ $h / 2$, на котором последовательность $f_{n}(\tau)$ равномерно стремится к нулю. Выберем число $n_{0}$ так, что при $n>n_{0}$ для $\tau \in \Theta_{1}$ выполнено неравенство $f_{n}(\tau)<1 / 4$.

При соответствии П из леммы 3 построенной выше функции $u$ ставится в соответствие семейство последовательностей $U(\tau) \in l_{p}$, таких, что $U(\tau)(k)=$ 
$(N+1)^{-1 / p} \lambda_{\nu}^{-k} h^{-1 / p} \eta$ при $N_{1} \leqslant k \leqslant N_{1}+N$ и $U(\tau)(k)=0$ при остальных $k$. При этом $\|U(\tau)\|=h^{-1 / p}$.

Отметим, что если $\|w(\tau)\|>2 h^{-1 / p}$, то $\|U(\tau)-w(\tau)\|>h^{-1 / p}$.

Пусть теперь $\|w\| \leqslant 2 h^{-1 / p}$. Оценим снизу $\|U(\tau)-w(\tau)\|$ в этом случае. Воспользовавшись тем, что $w(\tau)=w(\tau, \xi) \in W(\tau)$ для некоторого $\xi$, и равенством (16), получаем, что $|\xi| \leqslant 2(\phi(\tau, \xi))^{-1} h^{-1 / p}$.

Пусть $N_{1}>n_{0}$. Тогда для $w(\tau, \xi) \in W(\tau)$ и $\tau \in \Theta_{1}$

$$
\left(\sum_{k>N_{1}}|w(\tau, k)|^{p}\right)^{1 / p}=\left[\phi(\tau, \xi)-\phi_{N_{1}}(\tau, \xi)\right]|\xi| \leqslant 2 f_{N_{1}}(\tau) h^{1 / p} \leqslant \frac{1}{2} h^{-1 / p} .
$$

Поэтому

$$
\begin{aligned}
\|U(\tau)-w(\tau)\| & \geqslant\left(\sum_{k>N_{1}}|U(\tau, k)-w(\tau, k)|^{p}\right)^{1 / p} \\
& \left.\geqslant\left|\left(\sum_{k>N_{1}} \mid U(\tau, k)\right)\right|^{p}\right)^{1 / p}-\left(\sum_{k>N_{1}}|w(\tau, k)|^{p}\right)^{1 / p} \mid \geqslant \frac{1}{2} h^{-1 / p} .
\end{aligned}
$$

Таким образом, для всех $w(\tau, \xi) \in W(\tau)$ выполнено $\|U(\tau)-w(\tau, \xi)\| \geqslant \frac{1}{2} h^{-1 / p}$.

Отсюда получаем, что

$$
\begin{aligned}
\|u-w\| & \geqslant\left(\int_{\Theta}\left(\sum_{k>N_{1}}|U(\tau, k)-w(\tau, k)|^{p}\right) d \tau\right)^{1 / p} \\
& \geqslant\left(\int_{\Theta_{1}}\left(\sum_{k>N_{1}}|U(\tau, k)-w(\tau, k)|^{p}\right) d \tau\right)^{1 / p} \\
& \geqslant\left(\int_{\Theta_{1}}\|U(\tau)-w(\tau)\|^{p} d \tau\right)^{1 / p} \geqslant \frac{1}{2}\left(\mu\left(\Theta_{1}\right)\right)^{1 / p} h^{-1 / p} \geqslant \frac{1}{4},
\end{aligned}
$$

а это и требовалось. Утверждение доказано.

3. Пусть образ оператора $I-B$ замкнут и по доказанному выполнено условие локальной гиперболичности. Докажем, что выполнено условие $d(\tau)=$ const.

Для измеримого подмножества $M \subset \Theta$ ненулевой меры пространство $L_{p}\left(M ; l_{p}\right)$ естественно вложено в $L_{p}\left(\Theta ; l_{p}\right)$. Это подпространство и его дополнение $L_{p}\left(\Theta \backslash M ; l_{p}\right)$ инвариантны относительно действия оператора $B$, и определено сужение $B_{M}: L_{p}\left(M ; l_{p}\right) \rightarrow L_{p}\left(M ; l_{p}\right)$ оператора $B$ на это подпространство. Поэтому образ оператора $I-B_{M}$ является замкнутым.

Предположим, что $d(\tau) \neq$ const. Пусть $d_{0}=\min d(\tau)$. Так как функция $d(\tau)$ является полунепрерывной сверху, множество $\left\{\tau: d(\tau)=d_{0}\right\}$ открыто. Рассмотрим в качестве $M$ один из интервалов $\left(\tau_{1}, \tau_{2}\right)$, составляющих это множество. В граничной точке $\tau_{1}$ имеем $d\left(\tau_{1}\right)>d_{0}$.

Выберем ортонормированные базисы $e_{j}(\tau), j=1, \ldots, d_{0}$, в подпространствах $V_{\tau}, \tau \in M$, непрерывно зависящие от $\tau$, и построим по ним проекторы $P(\tau)$ вида (10). Заметим, что, в отличие от рассуждения из части 1 доказательства теоремы, эти операторы определены не на отрезке, а на интервале $M$, но в силу утверждения леммы 4 о равномерной непрерывности проекторы $P(\tau), \tau \in M$, 
ограничены в совокупности и задают ограниченный проектор $P_{M}$ в $L_{p}\left(M, l_{p}\right)$ на ядро оператора $I-B_{M}$.

Выберем также базис в слое $V_{\tau_{1}}$ и построим по нему аналогично проектор $P\left(\tau_{1}\right)$.

Подпространство $\operatorname{Ker} P_{M}=\operatorname{Im}\left(I-P_{M}\right)$ является дополнением к $\operatorname{Ker}\left(I-B_{M}\right)$, и оператор $I-B_{M}$ биективно отображает Ker $P_{M}$ на замкнутое подпространство $\operatorname{Im}\left(I-B_{M}\right)$. Поэтому существует такая постоянная $C>0$, что выполнена оценка снизу $\left\|\left(I-B_{M}\right) u\right\| \geqslant C\|u\|, u \in \operatorname{Ker} P_{M}$. Тогда аналогичная оценка

$$
\|(I-B(\tau)(I-P(\tau)) u\|\geqslant C\|(I-P(\tau)) u \|
$$

выполнена для почти всех $\tau \in M$, а в силу непрерывности по $\tau$ - для всех $\tau$.

Векторы базиса $e_{j}(\tau)$ могут не иметь предела при $\tau \rightarrow \tau_{1}$. Более того, примеры показывают, что может не существовать предел подпространств $V_{\tau}$ при $\tau \rightarrow \tau_{1}$ и соответственно может не существовать предел проекторов $P_{\tau}$. Однако $V_{\tau}$ можно рассматривать как подпространства одного конечномерного пространства $\mathbb{C}^{m}$. Из конечномерности следует, что найдется последовательность $t_{k} \in M, t_{k} \rightarrow \tau_{1}$, такая, что существуют пределы $e_{j}\left(t_{k}\right)$ при $k \rightarrow \infty$, и тогда эти пределы образуют базис в некотором $d_{0}$-мерном подпространстве из $V_{\tau_{1}}$. Из равномерной непрерывности отображения $J$ следует, что при $\tau \rightarrow \tau_{1}$ существует $\lim P\left(t_{k}\right):=\widetilde{P}$, причем $\widetilde{P}$ не совпадает с $P\left(\tau_{1}\right)$, а является проектором на некоторое собственное подпространство в $\operatorname{Ker}\left(I-B\left(\tau_{1}\right)\right)$. Поэтому существует ненулевой вектор $v$ из $\operatorname{Ker}\left(I-B\left(\tau_{1}\right)\right)$, такой, что $\widetilde{P} v=0$. Переходя к пределу при $t_{k} \rightarrow \tau_{1}$ в $(17)$, получаем оценку $\|\left(I-B\left(\tau_{1}\right)(I-\widetilde{P}) u\|\geqslant C\|(I-\widetilde{P}) u \|\right.$ для предельного оператора. Однако для построенного выше ненулевого вектора $v$ имеем $\left(\left(I-B\left(\tau_{1}\right)\right)(I-\widetilde{P}) v=\left(I-B\left(\tau_{1}\right)\right) v=0\right.$, что противоречит последней оценке. Теорема доказана.

\section{§5. Существенные спектры оператора взвешенного сдвига}

Динамические существенные спектры линейного расширения $\beta$ определим как множества чисел $\lambda$, при которых нарушены некоторые из рассмотренных свойств линейного расширения $\beta_{\lambda}$ из спектрального семейства $(5)$, а именно $\sigma_{F^{ \pm}}(\beta)$ есть множество чисел $\lambda$, при которых $\beta_{\lambda}$ не является \pm -полугиперболическим, $\sigma_{K}(\beta)=\sigma_{F^{+}}(\beta) \cap \sigma_{F^{-}}(\beta), \sigma_{F}(\beta)=\sigma_{F^{+}}(\beta) \cup \sigma_{F^{-}}(\beta)$ и $\sigma_{G}(\beta)-$ множество чисел $\lambda$, при которых $\beta_{\lambda}$ не является когерентно локально гиперболическим.

Из теоремы 1 в такой терминологии вытекает

Tеорема 2. Существенные спектры оператора $B$ совпадают $c$ соответствующими динамическими существенными спектрами линейного расширения $\beta$, причем $\sigma(B)=\sigma_{F}(B)$.

Можно получить некоторую дополнительную информацию о динамических, а следовательно, и об операторных существенных спектрах. Пусть числа $r_{j}(0)$ и $m_{j}(0)$ те же, что в лемме, и пусть $r_{k}(1)$ и $m_{k}(1), k=1, \ldots, l(1)$, суть аналогичные числа для матрицы $a(1)$.

Положим $r_{0}(0)=r_{0}(1)=0, r_{l(0)+1}(0)=r_{l(1)+1}(1)=+\infty$, и пусть $R_{j}(x)=$ $\left\{\lambda: r_{j-1}(x)<|\lambda|<r_{j}(x)\right\}, x=0$ или $x=1, R_{j, k}=R_{j}(0) \cap R_{k}(1)$. Среди 
множеств $R_{j, k}$ могут быть пустые, но имеется ровно $\nu-1$ непустых, где $\nu-$ число различных среди чисел $r_{j}(0)$ и $r_{k}(1), \nu \leqslant l(0)+l(1)$.

Согласно утверждению теоремы 1 , окружности $|\lambda|=r_{j}(0)$ (резонансные окружности первого типа) и $|\lambda|=r_{k}(1)$ (резонансные окружности второго muna) принадлежат существенному спектру $\sigma_{G}(B)$. Множества $R_{j, k}$ представляют собой компоненты связности дополнения к объединению резонансных окружностей на комплексной плоскости, и для каждого $\lambda$, не принадлежащего резонансным окружностям, однозначно определены числа $j=j(\lambda)$ и $k=k(\lambda)$, такие, что $\lambda \in R_{j, k}$.

Множества $R_{0,0}$ (внутренний круг) и $R_{l(0)+1, l(1)+1}$ (внешность относительно резонансной окружности с наибольшим радиусом) принадлежат резольвентному множеству оператора. Остальные (непустые) множества $R_{j, k}$ являются кольцами.

ТЕОрема 3. 1) Для каждого из (непустых) колеи $R_{j, k}$ все его точки имеют один и тот же спектральный тип, т.е. все они принадлежат одним и тем же существенным спектрам либо все являются регулярными значениями.

2) Если $m_{j}(0) \neq m_{k}(1)$ и колъцо $R_{j, k}$ непусто, то оно принадлежит спекmpy.

3) В крайних кольцах $R_{j, k}$ (когда один из индексов принимает наименъшее или наибольшее значение) оператор $\lambda I-B$ односторонне обратим. А именно, для нерезонансных значений $\lambda$ при выполнении условия

$$
r_{1}(0)<|\lambda|<r_{1}(1) \quad \text { или } \quad r_{l(0)}(0)<|\lambda|<r_{l(1)}(1)
$$

оператор $\lambda I-B$ обратим слева, но необратим, а при выполнении условия

$$
\left.r_{1}(0)>|\lambda|>r_{1}(1) \quad \text { или } \quad r_{l(0)}(0)>|\lambda|>r_{l(1)}(1)\right)
$$

оператор $\lambda I-B$ обратим справа, но необратим.

ДоказАтельство. 1) Согласно лемме 2 , для всех $\lambda \in R_{j}(0)$ локально устойчивые в точке 0 подрасслоения для линейных расширений $\beta_{\lambda}$ совпадают. Аналогично, для $\lambda \in R_{k}(1)$ совпадают подрасслоения, локально устойчивые в точке 1. Поэтому для всех $\lambda \in R_{j, k}$ соответствующие функции $d(\tau)$ и $d^{*}(\tau)$ совпадают и такие $\lambda$ имеют одинаковый спектральный тип.

2) Так как $\operatorname{ind}(\lambda I-B(\tau))=m_{j}(0)-m_{k}(1) \neq 0$, операторы $\lambda I-B(\tau)$ необратимы и $\lambda$ является спектральным значением.

3) В указанных кольцах одно из двух устойчивых подрасслоений (в точке 0 и в точке 1) для $\beta$ или для $\beta^{*}$ нулевое и оператор $\lambda I-B$ односторонне обратим.

ПримеР 1. Для оператора вида (1) с постоянным исходным коэффициентом $a_{0}(x) \equiv a_{0}(0)$ локально устойчивые подрасслоения имеют вид произведения (слои не зависят от $x$ ) и локальные гиперболические структуры всегда когерентны. Поэтому $\sigma_{G}(B)=\sigma_{K}(B)$ и эти спектры состоят только из резонансных окружностей. Кроме того, если в этом примере выполнено условие $\alpha(x)<x$, то для колец, заключенных между резонансными окружностями, $d_{\lambda}(\tau)=0$, a $d_{\lambda}^{*}(\tau)=r(\lambda)-\nu(\lambda)$, где $r(\lambda)$ есть число (с учетом кратности) собственных значений матрицы $a$, удовлетворяющих неравенству $\left|\lambda_{j}\right| \alpha^{\prime}(0)<|\lambda|$, а $\nu(\lambda)$ - число собственных значений, удовлетворяющих неравенству $\left|\lambda_{j}\right| \alpha^{\prime}(1)<|\lambda|$. Поэтому $\sigma_{F}+$ состоит только из резонансных окружностей, а каждое из указанных колец либо принадлежит резольвентному множеству, либо принадлежит $\sigma_{F^{-}}$. 
ПримеР 2. Пусть $m=1$, т. е. рассмотрим скалярный случай. Тогда спектр состоит из одного кольца с радиусами $|a(0)|$ и $|a(1)|$ и в силу утверждения 3$)$ теоремы 3 для внутренних точек этого кольца оператор $\lambda I-B$ односторонне обратим. Это утверждение было ранее получено Ю. И. Карловичем (см. [15]).

Пример 3. Пусть $m=2, A(x)=\operatorname{diag}\left\{\lambda_{1}(x), \lambda_{2}(x)\right\}$ - диагональная матрица, причем $\left|\lambda_{1}(0)\right|<\left|\lambda_{1}(1)\right|<\left|\lambda_{2}(0)\right|<\left|\lambda_{2}(1)\right|$, и пусть при $x \in \Theta$

$$
V(x)=\left(\begin{array}{cc}
\cos (2 \pi(x-t) / h) & \sin (2 \pi(x-t) / h) \\
-\sin (2 \pi(x-t) / h) & \cos (2 \pi(x-t) / h)
\end{array}\right),
$$

где $h=t-\alpha(t)$, и $V(x)$ есть единичная матрица при остальных $x$. Рассмотрим оператор (1) с приведенным коэффициентом $a(x)=A(x) V(x)$. Тогда спектр $\sigma(B)$ есть кольцо $\left|\lambda_{1}(0)\right| \leqslant|\lambda| \leqslant\left|\lambda_{2}(1)\right|$, причем при $\left|\lambda_{1}(0)\right|<|\lambda|<\left|\lambda_{1}(1)\right|$ образ оператора $\lambda I-B$ замкнут, $\operatorname{Ker}(\lambda I-B)=0$ и оператор обратим слева, при $\left|\lambda_{1}(0)\right| \leqslant|\lambda| \leqslant\left|\lambda_{2}(0)\right|$ образ этого оператора незамкнут и при $\left|\lambda_{2}(0)\right|<|\lambda|<$ $\left|\lambda_{2}(1)\right|$ образ совпадает со всем пространством и оператор обратим справа.

Приведенный пример 3 показывает, что все логически возможные случаи, описанные в теореме, реализуются для операторов указанного вида с произвольными коэффициентами. Примеры 1 и 2 показывают, что для специальных подклассов, которые рассматривались ранее, имеет место вырождение: число возможных качественно различных вариантов уменьшается, так как некоторые из существенных спектров совпадают.

\section{ЛИТЕРАТУРА}

1. Аносов Д. В. Об аддитивном гомологическом уравнении, связанном с эргодическим поворотом окружности. Изв. АН СССР, сер. матем., 37, №2, 1259-1274 (1973).

2. Антоневич А. Б. Об операторах, порожденных линейными расширениями диффеоморфизмов. ДАН СССР, 243, №4, 825-828 (1978).

3. Антоневич A. Б. Линейные функциональные уравнения. Операторный подход. Университетское, Минск, 1988.

4. Антоневич А. Б., Лебедев А. В. Функциональные и функционально-операторные уравнения. $C^{*}$-алгебраический подход. Труды С.-Петербург. матем. о-ва, 6, 34140 (1998).

5. Бронштейн И. У. Неавтономные динамические системы. Штиинца, Кишинев, 1984.

6. Горин E. A. Как выглядит спектр эндоморфизма диск-алгебры. Зап. науч. семин. ЛОМИ, 126, 55-68 (1983).

7. Китовер A. K. О спектре автоморфизмов с весом и теореме Камовица-Шейнберга. Функц. анализ и его прил., 13, вып. 1, 70-71 (1979).

8. Латушкин Ю. Д., Степин А. М. Операторы взвешенного сдвига и линейные расширения динамических систем. УМН, 46, вып. 3 (278), 85-143 (1991).

9. Antonevich A., Lebedev A. Functional differential equations: I. $C^{*}$-theory. Longman Scientific \& Technical, Harlow, 1994.

10. Antonevich A. B., Belitskii G. The essential spectrums of a weighted shift operator in the spaces of continuous and differentiable functions. In: Spectral and evolutionary problems, Vol. 8, Tavria Publ., Simferopol, 1998, pp. 3-12.

11. Belitskii G., Bykov N. Spaces of cohomologies associated with linear functional equations. Ergodic Theory Dynam. Systems, 18, 343-356 (1998). 
12. Belitskii G., Lyubich $Y u$. On the normal solvability of cohomological equations on compact topological spaces. In: Operator Theory: Advances and Applications, Vol. 103, 1998, pp. $75-87$.

13. Chicone C., Latushkin Yu. Evolution Semigroups in Dynamical Systems and Differential Equations. Amer. Math. Soc., Providence, RI, 1999.

14. Karlovich A. Yu., Karlovich Y. I. One-sided invertibility of binomial functional operators with a shift in rearrangement-invariant spaces. Integral Equations Operator Theory, 42, 201-228 (2002).

15. Kravchenko V. G., Litvinchuk G. S. Introduction to the Theory of Singular Integral Operators with Shift. Math. Appl., Vol. 289, Kluwer, 1994.

Белорусский государственный университет

Университет в Белостоке

Поступило в редакцию

e-mail: antonevich@bsu.by

3 мая 2003 г. 